\title{
Determinants of altered quality of life in patients with sleep-related breathing disorders
}

\author{
E. Sforza*, J.P. Janssens ${ }^{\#}$, T. Rochat ${ }^{\#}$, V. Ibanez*
}

Determinants of altered quality of life in patients with sleep-related breathing disorders. E. Sforza, J.P. Janssens, T. Rochat, V. Ibanez. (C) ERS Journals Ltd 2003.

ABSTRACT: Recent reports have suggested that altered quality of life and well-being are reported by patients with sleep-related breathing disorders (SRBD). There seems to be no data available in the literature on factors underlying these behavioural consequences. In this study, health-related quality of life (HRQL) scores were examined in SRBD patients in order to establish which factors are implicated in these disturbances.

The study group consisted of 130 patients: 49 snorers and 81 patients with obstructive sleep apnoea. The Medical Outcome Survey Short Form-36 questionnaire was administered the morning after the sleep study and scores for the eight dimension scores were obtained. Patient's data were compared to normative sex- and age-matched data.

In comparison with normal values, scores for all HRQL dimensions were decreased in SRBD patients, with a greater impact on subscores for "vitality", "physical role", "social functioning", "mental health" and "role emotional" dimensions. While impairment in physical function was mostly influenced by sleep stage and obesity, subjective daytime sleepiness mainly affected the other dimensions.

The authors conclude that the altered health-related quality of life of sleep-related breathing disorder patients is a multifactorial phenomenon depending on the interaction of sleep stages, daytime sleepiness and obesity, with no significant contribution of sleep fragmentation, hypoxaemia and apnoea recurrence.

Eur Respir J 2003; 21: 682-687.
*Sleep Laboratory, Division of Neurophysiology, Dept of Psychiatry, and ${ }^{\#}$ Division of Pneumonology, Geneva University Hospital, Geneva, Switzerland.

Correspondence: E. Sforza

Unité de Neurophysiologie

Laboratoire de sommeil

Département de Psychiatrie

Hôpital Belle Idée

2 Chemin du Petit Bel Air

Ch 1225 Chêne Bourg

Genève

Switzerland

Fax: 41223055343

E-mail: Emilia.Sforza@hcuge.ch

Keywords: Daytime sleepiness

quality of life

sleep continuity

sleep-related breathing disorders

Received: September 212002

Accepted after revision: November 212002
Sleep-related breathing disorders (SRBD) are common disorders, which affect $\geqslant 2-4 \%$ of the general population and are characterised by repetitive pharyngeal collapse during sleep, inducing sleep fragmentation and nocturnal hypoxaemia. These two factors have been suggested as causal factors of the excessive daytime sleepiness and neuropsychological and cognitive disturbances affecting the daytime functioning of these patients. Several studies have demonstrated that a large number of patients with SRBD may also have altered health-related quality of life (HRQL) scores, using either generic questionnaires, such as the Nottingham Health Profile $[1,2]$ or the Medical Outcome Survey Short Form (SF)-36 [3], or disease-specific questionnaires, such as the Calgary Sleep Apnea Quality of Life [4, 5]. These all showed impairment more frequently in vitality and social functioning [6]. Despite the frequent association between SRBD and altered daily functioning and well-being [7, 8], and the importance of altered quality of life in SRBD [6], no data are available in the literature concerning the mechanisms underlying these psychological disturbances. This could be due, at least in part, to the fact that quality of life scores are not routinely used by clinicians to detect the impact of the sleep disorder on the well-being of their patients [9] and also to the difficulty in defining which parameters better reflect a clinically meaningful improvement after treatment [10]. The current hypothesis to explain the altered quality of life stresses the role of excessive daytime sleepiness [6-8], since increased sleepiness may affect the general perception of emotional [11] and physical health and impairs social interaction [12]. This hypothesis is based on clinical studies showing that efficacious treatment with nasal continuous positive airway pressure (nCPAP) significantly improves the behavioural consequences of SRBD [13-16] and is associated with a significant reduction in daytime sleepiness, an increase in energy and an improvement in emotional perceptions.

Despite the fact that fatigue, sleepiness and tiredness may all influence the patient's quality of life, to the current authors' knowledge, the critical factors, which most affect the perception of emotional and physical health, remain unknown. The aim of the present study was two-fold: first, to ascertain which scores of HRQL are most affected in a large sample of patients with SRBD; and secondly, if, as expected, a decrease in some of the SF-36 scores is documented, to determine whether daytime sleepiness, sleep fragmentation, recurrence of breathing disorders or hypoxaemia would better predict the degree of changes in quality of life measures and behavioural morbidity in these patients.

\section{Methods}

\section{Subjects}

During an 18-month period (December 2001-June 2002), all consecutive patients referred to the authors' sleep laboratory for evaluation of snoring, witnessed apnoeas and daytime sleepiness were included. A total of 177 patients were identified from the polysomnographical study register. Exclusion criteria included the following: 1) previous treatment for sleep apnoea with nCPAP, corrective upper airway 
surgery or a mandibular advancement device $(\mathrm{n}=10) ; 2)$ presence of other sleep disorders, such as narcolepsy or periodic leg movements $(n=12)$; 3) refusal to perform maintenance wakefulness test (MWT) $(n=15)$; and 4) presence of a mental, neurological or physical impairment severe enough to preclude their ability to read and complete the questionnaires $(n=10)$. Of the original sample, 130 patients $(28$ females and 102 males) aged $53.6 \pm 0.9$ yrs (mean \pm SEM), with a mean body mass index (BMI) of $31.8 \pm 0.6 \mathrm{~kg} \cdot \mathrm{m}^{-2}$ and a mean neck circumference of $41.4 \pm 0.3 \mathrm{~cm}$, fulfilled the inclusion criteria. Patients were informed that some of the collected data would be used for research purposes and they gave written informed consent.

\section{Nocturnal sleep studies}

Polysomnography included seven electroencephalograms (EEG; F3-A2, C3-A2, O1-A2, F4-A1, C4-A1, O2-A1, CZA1), right and left electro-oculograms and one electromyogram (EMG) of chin muscles for conventional sleep staging. Respiratory airflow was monitored with a nasal cannula connected to a pressure transducer (Protech2; Protech, Minneapolis, MN, USA), thoracic and abdominal respiratory movements with piezoelectric strain gauges, and tracheal sound by microphone. Arterial oxygen saturation $\left(\mathrm{Sa}, \mathrm{O}_{2}\right)$ was continuously measured with a finger oximeter.

Sleep was scored using the criteria of RECHTSCHAFFEN and KALES [17] for epochs of $20 \mathrm{~s}$ by a scorer experienced in the use of standard guidelines. As indices of sleep fragmentation, the number of arousals and awakenings were defined, as well as the number of sleep state transitions. Macroarousals were scored according to American Sleep Disorders Association criteria [18] as abrupt shifts in EEG frequency, irrespective of chin EMG changes during nonrapid eye movement sleep (NREM) but associated with a concurrent EMG increase in rapid eye movement (REM) sleep. Respiratory events were scored using standard criteria. Hypopnoeas were defined as $\geqslant 50 \%$ reduction in airflow from the baseline value lasting $\geqslant 10 \mathrm{~s}$ and associated with $3 \%$ desaturation or an arousal. Apnoeas were defined as the absence of airflow on the nasal cannula lasting for $>10 \mathrm{~s}$. The apnoea/hypopnoea index (AHI) was established as the ratio of the number of apnoeas and hypopnoeas per hour of sleep. As indices of nocturnal hypoxaemia, the mean $\mathrm{Sa}, \mathrm{O}_{2}$ and the minimal value recorded during sleep $\left(\mathrm{Sa}, \mathrm{O}_{2} \mathrm{~min}\right)$ were considered.

For descriptive analysis, an AHI cut-off point of 15 was used to stratify patients into the following two groups. Snorers: AHI $\leqslant 15 \quad(n=49) ;$ and obstructive sleep apnoea (OSA) patients: AHI $>15(\mathrm{n}=81)$.

\section{Daytime sleepiness evaluation}

To evaluate the level of sleepiness, patients completed the Epworth Sleepiness Scale (ESS) on the morning after the nocturnal polysomnography. The ESS is a validated questionnaire containing eight items that ask for self-reported probability of dozing in a variety of situations. The dozing probability ranges from 0 (never) to 3 (high probability). Normal values range between 2 and 10 , with scores $>10$ indicating daytime sleepiness [19]. During the MWT, the subjective level of sleepiness was assessed using the Stanford Sleepiness Scale (SSS) and the Visual Analogue Scale (VAS). The SSS [20] contains seven statements describing different levels of current alertness, ranging from 1 "feeling alert and vital" to 7 "almost in reverie, lost struggle to remain awake". The patient has to choose the most appropriate description of their sleepiness level. The VAS [21] is a 10-cm line with the inscription "very sleepy" on the left and "very alert" on the right. Subjects were instructed to draw a vertical bar across the line at the point corresponding to their subjective feeling of alertness.

Objective daytime sleepiness was assessed with the MWT according to standard criteria [22]. This was done by asking the patients to sit in a quiet, dark room and to try to stay awake during five sessions scheduled at 09:00, 11:00, 13:00, 15:00 and 17:00 h. All tests were terminated 15 min after sleep onset or after 40 min without sleep, and mean sleep latency was calculated by averaging the latencies to sleep for the five naps.

\section{Anxiety, depression and quality of life questionnaires}

All subjects were administered two previously validated questionnaires on the morning after the sleep study. Anxiety and depression were measured by means of the Hospital Anxiety and Depression Scale (HAD) [23]. The HAD is a selfadministered rating scale consisting of two subscales, one scoring for anxiety and one for depression, each containing seven items on a 4-point Likert scale (range 0-3). HRQL was measured using the French validated version of the SF-36 [24]. The SF-36 is a self-administered generic questionnaire that assesses health status as well as the subjective perception of limitation in function and health by analysing eight dimensions: physical functioning, social functioning, physical role, emotional role, general health, mental health, vitality, and body pain. Scores were coded, summed and transformed into a scale from 0 (worst possible health) to 100 (best possible health) for each dimension. The SF-36 scores of the patient groups were compared to normative data reported in the literature [25], individually matched for age and sex.

\section{Statistical analyses}

Values for SF-36 and HAD scores were non-normally distributed so that mean values were expressed as median (95\% confidence interval $(\mathrm{CI})$ ), while polysomnographical and anthropometric values were expressed as means \pm SEM.

Differences between controls, snorers and OSA patients were evaluated using a nonparametric test (Mann-Whitney U-test) with Bonferroni's corrections for multiple comparisons. The differences were considered significant if they had $p$-values of $<0.05$. Bivariate correlation analysis using Spearman's rank correlation was used to identify variables correlated with the HAD and the SF-36 scores, and multivariate regression analysis was performed to define the contribution of anthropometric, diurnal and nocturnal variables in explaining SF-36 scores.

\section{Results}

\section{Characteristics of the patients}

Table 1 shows mean \pm SEM values for clinical, anthropometric and polysomnographical parameters among the analysed patients stratified according to an AHI cut-off value of 15 . The two groups were similar in age and BMI, whereas neck circumference was greater among patients with OSA. With regard to polysomnographical parameters, table 1 reveals statistically significant differences between the groups. The OSA group had lower nocturnal $\mathrm{Sa}, \mathrm{O}_{2}$ and slow wave sleep (SWS) and a higher respiratory disturbances index, a 
Table 1. - Anthropometric, clinical and polysomnographical data of snorers and obstructive sleep apnoea (OSA) patients

\begin{tabular}{|c|c|c|c|c|}
\hline Characteristics & Total & Snorers & OSA & p-value ${ }^{\#}$ \\
\hline Subjects $n$ & 130 & 49 & 81 & \\
\hline Age yrs & $53.6(0.9)$ & $51.9(1.5)$ & $54.7(1.1)$ & NS \\
\hline $\mathrm{BMI} \mathrm{kg} \cdot \mathrm{m}^{-2}$ & $31.8(0.6)$ & $31.5(1.0)$ & $32.0(0.7)$ & NS \\
\hline Neck circumference $\mathrm{cm}$ & $41.4(0.3)$ & $39.8(0.4)$ & $42.3(0.4)$ & 0.001 \\
\hline AHI $n \cdot h^{-1}$ & $32.2(2.4)$ & $8.4(0.6)$ & $46.6(2.8)$ & 0.001 \\
\hline Minimal $\mathrm{Sa}, \mathrm{O}_{2} \%$ & $78.3(0.71)$ & $82.8(0.7)$ & $75.5(0.9)$ & 0.001 \\
\hline Mean $\mathrm{Sa}, \mathrm{O}_{2} \%$ & $91.8(0.2)$ & $92.6(0.3)$ & $91.3(0.3)$ & 0.001 \\
\hline Stage $1 \%$ & $18.3(0.7)$ & $14.5(0.7)$ & $20.7(1.0)$ & 0.001 \\
\hline Stage $2 \%$ & $52.9(0.9)$ & $51.8(1.2)$ & $53.7(1.2)$ & NS \\
\hline Stages $3-4 \%$ & $12.0(0.7)$ & $15.0(0.9)$ & $10.2(0.9)$ & 0.001 \\
\hline Stage REM \% & $17.1(0.5)$ & $19.3(0.8)$ & $15.8(0.5)$ & 0.001 \\
\hline TTS min & $404.5(5.7)$ & $417.0(8.4)$ & $396.1(7.4)$ & NS \\
\hline WASO min & $101.7(4.9)$ & $92.6(7.1)$ & $107.3(6.5)$ & NS \\
\hline $\mathrm{SE} \%$ & $76.4(1.1)$ & $77.6(2.1)$ & $75.7(1.2)$ & NS \\
\hline Awakenings $\mathrm{n}$ & $17.7(1.1$ & $16.2(1.4)$ & $18.6(1.6)$ & NS \\
\hline Macroarousals n & $347.8(15.9)$ & $255.1(12.5)$ & $404.6(22.2)$ & 0.001 \\
\hline SSS & $2.6(0.1)$ & $2.5(0.1)$ & $2.7(0.1)$ & NS \\
\hline ESS & $10.6(0.4)$ & $10.5(0.6)$ & $10.6(0.6)$ & NS \\
\hline VAS & $2.6(0.2)$ & $2.4(0.2)$ & $2.7(0.2)$ & NS \\
\hline MWT min & $22.3(1.1)$ & $25.1(1.6)$ & $20.5(1.4)$ & 0.04 \\
\hline
\end{tabular}

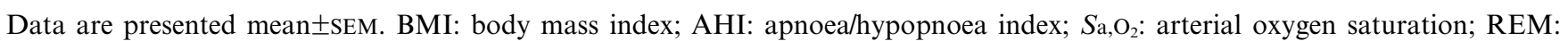
rapid eye movement; TTS: total sleep time; WASO: wake after sleep onset; SE: sleep efficiency; SSS: Stanford Sleepiness Scale; ESS: Epworth Sleepiness Scale; VAS: Visual Analogue Scale; MWT: mean sleep latency at the Maintenance Wakefulness Test; NS: nonsignificant. "\#. Mann-Whitney U-test.

higher proportion of stage 1 NREM sleep and more arousals. During the day, subjective sleepiness, as assessed by SSS, VAS and ESS, was not significantly different between snorers and OSA patients. However, measurement of objective sleepiness revealed that patients with a higher AHI (OSA group) were those who had lower mean sleep latency at the MWT. Of the original sample, 59 patients had objective pathological sleepiness defined by a mean sleep latency of $<20$ min and 60 patients reported an ESS score of $>10$.

\section{Medical Outcome Survey Short Form-36 dimension} scores and Hospital Anxiety and Depression Scale scores

Figure 1 shows the absolute results of the patients' SF-36 scores. Statistical analysis revealed that SRBD patients had significantly worse mean scores in all dimensions when compared to normative data. The greatest differences were seen for "role physical", "vitality", "social functioning", "role emotional" and "mental health" $(\mathrm{p}<0.001)$ dimensions, followed by significant differences in the "physical function" and the "general health" scores. The lowest difference, although significant, was in the "bodily pain" dimension. Data relevant to the item bodily pain are not reported because they lack clinical relevance when discussing HRQL scores in SRBD.

Mean scores of the HAD questionnaire were within normal range both for the anxiety subscale (HAD-A; mean $(95 \% \mathrm{CI})$ $8.8(8.2-9.4)$ ) and for the depression subscale (HAD-D; 6.3 (5.5-7.0)). When applying the criteria [23] for detecting anxiety and depression (score $>10$ ), the incidences of clear cases were $20 \%$ and $13 \%$ for anxiety and depression, respectively.

Table 2 reports the absolute scores of the SF-36 and HAD scores in the two groups of patients with snoring and OSA. OSA patients had worse scores in the physical functioning, role physical, bodily pain and general health dimensions than snorers, but the differences did not reach statistical significance.

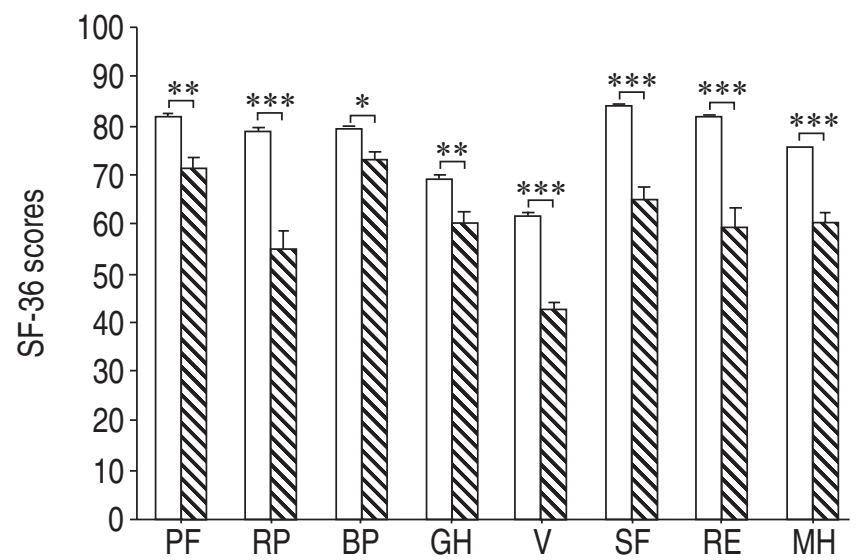

Fig. 1.-Histogram showing the Medical Outcome Survey Short Form (SF)-36 questionnaire scores for sleep-related breathing disorder patients $(\mathbb{\mathbb { Q }})$ compared with normative values $(\square)$. PF: physical function; RP: role physical; BP: bodily pain; GH: general health; V: vitality; SF: social functioning; RE: role emotional; $\mathrm{MH}$ : mental health. All measures of health were altered in the patients, mostly affecting vitality, physical and social functioning, and mental health. $*: \mathrm{p}<0.05 ; * *: \mathrm{p}<0.01 ; * * * \mathrm{p}<0.001$.

\section{Statistical analysis}

Results of a bivariate analysis (Spearman's rank correlation) of scores for seven dimensions of the SF-36 versus clinical and polysomnographical parameters and indexes of sleepiness are shown in table 3. BMI correlated negatively with all domains. $\mathrm{Sa}, \mathrm{O}_{2}$ min, amount of stage 1 and SWS were correlated mainly with physical functioning and role physical items, and to a lesser degree with general health and vitality scores. AHI and number of macroarousals were related to physical functioning. Subjective perception of daytime sleepiness (ESS, SSS, VAS) contributed significantly to lower scores for all seven domains. Conversely, objective measurements of daytime sleepiness (MWT) correlated positively with all HRQL scores, with the exception of role emotional. Interestingly, all 
Table 2.-Scores of the Medical Outcome Survey Short Form-36 questionnaire dimensions and Hospital Anxiety and Depression Scale (HAD) in the total group, snorers and obstructive sleep apnoea (OSA) patients

\begin{tabular}{|c|c|c|c|c|}
\hline & Total & Snorers & OSA & p-value ${ }^{\#}$ \\
\hline Physical functioning & $71.3(66.7-75.8)$ & $75.4(68.2-82.6)$ & $68.8(63.0-74.6)$ & NS \\
\hline Role physical & $55.2(48.2-62.2$ & $57.7(46.3-69.0)$ & $53.7(44.7-62.7)$ & NS \\
\hline Bodily pain & $79.1(70.2-86.0)$ & $80.5(75.7-85.2)$ & $74.7(70.9-78.4)$ & NS \\
\hline General health perception & $60.2(56.3-64.2)$ & $63.0(57.0-69.1)$ & $58.5(53.3-63.7)$ & NS \\
\hline Vitality & $42.3(38.5-46.0)$ & $41.1(35.4-46.9)$ & $43.0(37.9-48.0)$ & NS \\
\hline Social functioning & $65.1(60.5-69.7)$ & $65.0(57.8-72.2)$ & $65.2(59.1-71.3)$ & NS \\
\hline Role emotional & $59.5(52.1-66.9)$ & $57.8(45.1-70.5)$ & $60.5(51.2-69.8)$ & NS \\
\hline Mental health & $60.3(56.8-63.8)$ & $59.2(53.9-64.5)$ & $61.0(56.3-65.7)$ & NS \\
\hline HAD anxiety & $8.8(8.2-9.4)$ & $8.7(7.8-9.6)$ & $8.9(8.1-9.7)$ & NS \\
\hline HAD depression & $6.3(5.5-7.0)$ & $6.4(5.2-7.5)$ & $6.2(5.2-7.2)$ & NS \\
\hline
\end{tabular}

Data are presented as median (95\% confidence interval). ${ }^{*}$ : Mann-Whitney U-test.

Table 3. - Spearman's correlation coefficient between Medical Outcome Survey Short Form (SF)-36 scores, and diurnal and nocturnal parameters

\begin{tabular}{|c|c|c|c|c|c|c|c|}
\hline SF-36 measures & $\mathrm{PF}$ & $\mathrm{RP}$ & $\mathrm{GH}$ & V & SF & $\mathrm{RE}$ & MH \\
\hline Age & -0.10 & 0.12 & $0.19 *$ & 0.17 & $0.23^{\#}$ & 0.14 & $0.39^{\bullet}$ \\
\hline BMI & $-0.42^{\top}$ & $-0.24^{\#}$ & $-0.27^{\#}$ & $-0.25^{\#}$ & $-0.26^{\#}$ & $-0.24^{\#}$ & $-0.31^{\bullet}$ \\
\hline $\begin{array}{l}\text { Neck } \\
\quad \text { circumference }\end{array}$ & $-0.27^{\#}$ & -0.16 & -0.17 & -0.04 & -0.14 & -0.02 & -0.14 \\
\hline AHI & $-0.17 *$ & -0.09 & -0.12 & -0.01 & 0.05 & 0.04 & 0.12 \\
\hline Minimal $\mathrm{Sa}, \mathrm{O}_{2} \%$ & $0.36^{\top}$ & $0.19^{*}$ & $0.19^{*}$ & 0.09 & 0.09 & 0.05 & 0.03 \\
\hline Mean $S \mathrm{a}, \mathrm{O}_{2} \%$ & $0.30^{\curvearrowleft}$ & 0.08 & 0.10 & 0.06 & 0.09 & 0.07 & -0.02 \\
\hline Stage $1 \%$ & $-0.29^{\top}$ & $-0.18 *$ & -0.13 & $-0.19 *$ & -0.06 & -0.11 & 0.04 \\
\hline Stage $2 \%$ & 0.06 & -0.13 & 0.01 & 0.02 & 0.01 & -0.03 & 0.05 \\
\hline Stages $3-4 \%$ & $0.23^{\#}$ & $0.23^{\#}$ & $0.22 *$ & $0.22 *$ & 0.04 & 0.06 & 0.01 \\
\hline Stage REM \% & 0.02 & $0.18^{*}$ & 0.03 & 0.01 & -0.06 & 0.08 & -0.09 \\
\hline $\mathrm{SE} \%$ & 0.01 & 0.05 & -0.08 & -0.02 & 0.02 & 0.11 & -0.03 \\
\hline Awakeni & -0.06 & 0.05 & 0.09 & 0.05 & 0.08 & -0.01 & $0.17^{*}$ \\
\hline Macroarousal n & $-0.22^{*}$ & -0.15 & -0.14 & -0.13 & -0.01 & -0.04 & 0.15 \\
\hline SSS & $-0.32^{\top}$ & $-0.43^{\bullet}$ & $-0.36^{\circ}$ & $-0.49^{\top}$ & $-0.41^{\top}$ & $-0.43^{\top}$ & $-0.41^{\bullet}$ \\
\hline ESS & -0.14 & $-0.28^{\#}$ & -0.15 & $-0.39^{\top}$ & $-0.39^{\top}$ & $-0.29^{\#}$ & $-0.28^{\#}$ \\
\hline VAS & $-0.31^{\top}$ & $-0.47^{\bullet}$ & $-0.37^{\bullet}$ & $-0.53^{\circ}$ & $-0.47^{\bullet}$ & $-0.43^{\bullet}$ & $-0.42^{\circ}$ \\
\hline MWT min & $0.22 *$ & $0.21 *$ & $0.19 *$ & $0.25^{\#}$ & $0.19^{*}$ & 0.13 & $0.19^{*}$ \\
\hline HAD-A & $-0.26^{\#}$ & $-0.32^{\circ}$ & $-0.38^{\top}$ & $-0.37^{\bullet}$ & $-0.51^{\bullet}$ & $-0.55^{\bullet}$ & $-0.63^{9}$ \\
\hline HAD-D & $-0.45^{\top}$ & $-0.51^{\bullet}$ & $-0.54^{\top}$ & $-0.59^{\top}$ & $-0.59^{\top}$ & $-0.61^{\top}$ & $-0.66^{\circ}$ \\
\hline
\end{tabular}

PF: physical functioning; RP: role physical; GH: general health; $\mathrm{V}$ : vitality; SF: social functioning; RE: role emotional; $\mathrm{MH}$ : mental health; BMI: body mass index; AHI: apnoea/hypopnoea index; $\mathrm{Sa}_{\mathrm{a}} \mathrm{O}_{2}$ : arterial oxygen saturation; REM: rapid eye movement; SE: sleep efficiency; SSS: Stanford Sleepiness Scale; ESS: Epworth Sleepiness Scale; VAS: Visual Analogue Scale; MWT: mean sleep latency at the Maintenance Wakefulness Test; HAD-A: anxiety score of the Hospital Anxiety and Depression Scale (HAD) questionnaire; HAD-D: depression score of the HAD questionnaire. *: $\mathrm{p}<0.05 ;{ }^{\#}: \mathrm{p}<0.005$; : $\mathrm{p}<0.0001$.

scores estimating subjective and objective daytime sleepiness were significantly correlated with the energy and vitality dimension, one of the scores of the SF-36 questionnaires greatly impaired in the study patients.

A stepwise multiple regression analysis, including BMI, nocturnal parameters and indices of daytime sleepiness as independent variables and successive SF-36 scores as dependent variables, showed that BMI, amount of stage 1 and SWS, SSS and ESS made a significant contribution to SF-36 scores with a somewhat different relative contribution to the seven HRQL dimensions (table 4). For physical functioning it was found that a model including BMI, amount of stage 1 and SSS could explain $24 \%$ of the variance. Identical significant predictors were found for role physical dimension,
Table 4.-Results of multiple regression analysis for the Medical Outcome Survey Short Form (SF)-36 scores

\begin{tabular}{lccc}
\hline Dependent variable & Independent variable & $\mathrm{r}^{2}$ & $\mathrm{p}$-value \\
\hline Physical functioning & BMI & 0.15 & 0.001 \\
& Stage 1 & 0.21 & 0.002 \\
Role physical & SSS & 0.24 & 0.04 \\
& ESS & 0.06 & 0.02 \\
& BMI & 0.12 & 0.007 \\
General health & SWS & 0.15 & 0.04 \\
& SSS & 0.14 & 0.001 \\
Vitality & BMI & 0.18 & 0.02 \\
Social functioning & ESS & 0.15 & 0.001 \\
& ESS & 0.17 & 0.001 \\
Role emotional & BMI & 0.22 & 0.008 \\
& ESS & 0.09 & 0.001 \\
Mental health & BMI & 0.13 & 0.02 \\
& ESS & 0.09 & 0.001 \\
& BMI & 0.15 & 0.004 \\
\hline
\end{tabular}

BMI: body mass index; SSS: Stanford Sleepiness Scale; ESS: Epworth Sleepiness Scale; SWS: slow wave sleep.

ESS, BMI and amount of SWS, explaining 15\% of the variance. Subjective daytime sleepiness, as defined by the SSS and ESS scores, mostly contributed to the variance of general health $(14 \%)$, vitality $(15 \%)$ and social functioning $(17 \%)$ dimensions.

\section{Discussion}

The aim of the present study was to consider all factors, both diurnal and nocturnal, that may be involved in the effect of SRBD on health status. The large sample and the analysis of all potential factors implicated were expected to allow comparison of an adequate number of patients with different severity of disease, to lead to a better characterisation of the factors influencing the relationship between altered well-being and disease severity. As expected, compared to normative data, SRBD patients reported lower social functioning, more limitations in function due to physical and emotional reasons and a lowered sense of well-being with regard to mental health and energy. This predisposition seems to be independent of the AHI and nocturnal hypoxaemia, as demonstrated by the comparison of patients with and without OSA who shared similar HRQL impairment. The most interesting finding of this study was that while objective assessment of SRDB (hypoxaemia, AHI, sleep disruption and sleep fragmentation) appeared to have a small impact only on scores relative to physical functioning, obesity and daytime 
sleepiness contributed more significantly to impairment in all domains of the SF-36 questionnaire.

Despite the considerable attention that has been directed at understanding the factors influencing the well-being and health perception of patients with SRBD in recent years, to date understanding about the factors that mostly affect perception of physical and emotional health is limited. The results of this study show that the consequences of SRBD on HRQL may be considered as a multifactorial phenomenon, including sleep disruption, sleepiness and obesity, which are all implicated in different aspects of physical and mental health. A primary finding of the current study is that HRQL scores exploring physical functioning were related to factors different from those quantifying vitality, social functioning and mental health. In the study group, the physical function and the role physical were related to nocturnal parameters indicating sleep disruption, i.e. amount of stage 1 and SWS, with an additional influence of indices of daytime sleepiness and BMI. On the basis of these results, it is difficult to discriminate fully between the effects of breathing disorders on sleep structure and the effects of diurnal sleepiness, as the two factors may be interrelated [26]. However, assuming that the amount of SWS and stage 1 are indicative of sleep continuity and sleep restorative ability [27], the sleepdisturbing effects of the respiratory events accumulated during one night's sleep may lead to tiredness and fatigue [28], which may be reflected by a decrease in physical function. Although there were no statistically significant differences between snorers and OSA patients in the current study group, it was noted that lower physical function and role physical scores were reported by OSA patients, in whom a lower amount of SWS and a greater amount of stage 1 was present, suggesting that the greater limitation in physical function may be a consequence of the recurrence of apnoeas. This would mean that when patients report impairment in physical function, they may merely be unable to separate "tiredness", which is related to nonrestorative sleep, from an organic and physical impairment.

In line with previous results [3-6], the decline in vitality, social functioning and emotional dimensions was significantly related to diurnal sleepiness, as assessed by SSS, VAS, ESS questionnaires and MWT, with a greater contribution for scores assessing subjective sleepiness. This may suggest that some of the psycho-physiological consequences of SRBD do not reflect a general psychological and mood effect but rather the specific consequences of sleepiness and impaired alertness. This could suggest that the sleepiness reported by SRBD patients translates both the inability to stay awake, as measured by sleepiness questionnaires and MWT, as well as a subjective feeling of "loss of energy" [11, 28].

The design of this study not only allowed the determination of nocturnal and diurnal factors influencing the quality of life of SRBD patients but also allowed the authors to ascertain whether the decline in the patient's well-being may be related to factors others than sleep disorders. It was noted that some SF-36 scores showed a negative and significant relationship with the BMI (except bodily pain) and obesity contributed to SF-36 score variance in the patients in this study. Quality of life scores were reported to be lower in $40-60 \%$ of obese populations [29] with an impairment linearly related to BMI, and improving after weight loss. However, in obese patients, physical limitation is frequently related to bodily pain with a small affect on mental health, role emotional and social functioning. A point of discussion is whether the SF-36 score impairment found in the patients in this study can be interpreted as evidence not simply of the underlying sleep disorder but also in relation to obesity. This point seems to been resolved, at least partially, by the results showing that bodily pain, the SF-36 score reported in $56 \%$ of the obese population, was the only one not significantly different in the current group compared to normative data. Although this study does not allow us to draw any conclusions as to the relationship between obesity, SRBD and quality of life, the data support the hypothesis that the quality of life impairment in SRBD patients is more a marker of OSA and snoring than of obesity.

Although significant relationships do exist between HRQL dimensions and some indices of sleep continuity, BMI and sleepiness, the correlations are mostly in the moderate-to-low range. There are several possible explanations for the lack of a strong effect of these factors on quality of life in the patients examined in this study. First, HRQL scores were obtained by administration of a "generic" HRQL questionnaire, which may have lacked sensitivity in detecting SRBD-induced changes in HRQL. Thus, it could be suggested that scores obtained in this study did not reflect the extent of the specific changes in well-being that may have occurred in these patients, and it seems reasonable to assume that stronger correlations may be revealed when patients are examined with a disease-specific questionnaire, such as the Calgary Sleep Apnea Quality of Life Index [4, 5, 9]. Secondly, it can be hypothesised that the current patient sample, although large, may be not representative of the general SRBD population. It could be correctly argued that extensive studies in a larger sample of patients, with a wider AHI and sleepiness distribution, and using disease-specific measures of quality of life, could provide more information on the role of sleepiness, sleep disruption and obesity on the quality of life of SRBD patients.

In conclusion, through comparison of the quality of life scores of sleep-related breathing disorder patients with normative data, it was found that impairment was present in all Medical Outcome Survey Short Form-36 dimensions in patients with sleep-related breathing disorders, mostly affecting physical functioning, vitality, social functioning, role emotional and mental health dimensions. All dimensions (except bodily pain) were negatively related to body mass index. Furthermore, while the decrease in physical function was influenced by objective indices of sleep discontinuity and subjective sleepiness, the impairment of social functioning, emotional well-being and vitality were related to consequences of sleepiness. Even though the use of a self-administered questionnaire is problematic and health perception can be affected by environmental situations and individual motivation, the current data support previous reports [9] suggesting that the extensive use of quality of life questionnaires in clinical evaluation may allow a better definition of the behavioural consequences of sleep-related breathing disorders and may add more information on factors influencing the well-being of these patients.

Acknowledgements. The authors are grateful to B. Adjivon, B. Bertram, D. Grasset, A. Lalji and J. Paillard for their technical assistance.

\section{References}

1. Hunt SM, McEwan J, McKenna SP. Measuring health status: a new tool for clinicians and epidemiologists. $J$ R Coll Gen Pract 1985; 35: 185-188.

2. Engleman HM, Martin SE, Douglas NJ. Compliance with CPAP therapy in patients with sleep apnoea/hypopnoea syndrome. Thorax 1994; 49: 263-266.

3. Jenkinson C, Stradling J, Petersen S. Comparison of three measures of quality of life outcome in the evaluation of 
continuous positive airway pressure therapy for sleep apnoea. J Sleep Res 1997; 6: 199-204.

4. Flemons WW, Reimer MA. Development of a diseasespecific Health-related Quality of Life Questionnaire for sleep apnea. Am J Respir Crit Care Med 1998; 158: 494-503.

5. Flemons WW, Reimer MA. Measurement properties of the Calgary Sleep Apnea Quality of Life Index. Am J Respir Crit Care Med 2002; 165: 159-164.

6. Smith IE, Shneerson JM. Is the SF 36 sensitive to sleep disruption? A study in subjects with sleep apnoea. J Sleep Res 1995; 4: 183-188.

7. Gall R, Isaac L, Kryger M. Quality of life in mild obstructive sleep apnea. Sleep 1993; 16: S59-S61.

8. Veale D, Poussin G, Benes F, Pepin JL, Levy P. Identification of quality of life concerns of patients with obstructive sleep apnoea at the time of initiation of continuous positive airway pressure: a discourse analysis. Qual Life Res 2002; 11: 389-399.

9. Lacasse Y, Godbout C, Séries F. Health-related quality of life in obstructive sleep apnoea. Eur Respir J 2002; 19: 499-503.

10. Reimer M, Flemons WW. Measuring quality of life in disorders of sleep and breathing. Sleep Breathing 1999; 3: 139-145.

11. Sforza E, De Saint Hilaire Z, Pelissolo A, Rochat T, Ibanez V. Personality, anxiety and mood traits in patients with sleep-related breathing disorders: effect of reduced daytime alertness. Sleep Medicine 2002; 3: 139-145.

12. Weaver TE, Laizner AM, Evans LK, et al. An instrument to measure functional status outcomes for disorders of excessive sleepiness. Sleep 1997; 20: 835-843.

13. Sanner BM, Klewer J, Trumm A, Randerath W, Kreuzer I, Zidek W. Long-term treatment with continuous positive airway pressure improves quality of life in obstructive sleep apnoea syndrome. Eur Respir J 2000; 16: 118-122.

14. Lacasse Y, Godbout C, Séries F. Independent validation of the Sleep Apnoea Quality of Life Index. Thorax 2002; 57: 483-488.

15. Bennett LS, Barbour C, Langford B, Stradling JR, Davies RJO. Health status in obstructive sleep apnea. Am J Respir Crit Care Med 1999; 159: 1884-1890.

16. Flemons WW, Tsai W. Quality of life consequences of sleep-disordered breathing. J Allergy Clin Immunol 1997; 99: S750-S756.

17. Rechtschaffen A, Kales A. A manual of standardized terminology, technique and scoring system for sleep stages of human sleep. Los Angeles Brain Information Service. UCLA, Brain Information Institute, 1968.

18. American Sleep Disorders Association. The Atlas Task Force. EEG arousals: scoring rules and examples. Sleep 1992; 15: 174-184.

19. Johns MW. A new method for measuring daytime sleepiness: the Epworth Sleepiness Scale. Sleep 1991; 14: 540-545.

20. Hoddes E, Dement WC, Zarcone V. The development and use of the Stanford Sleepiness Scale. Psychophysiology 1972; 9: 150 .

21. Johnson LC. Daytime sleepiness in good sleepers: measurement and correlates. In: Broughton RJ, Ogilvie RD, eds. Sleep, arousal and performance. Boston, Birkhauser, 1992; pp. 220-229.

22. Doghramji K, Mitler MM, Sangal RB, et al. A normative study of the maintenance of wakefulness test (MWT). Electronceph Clin Neurophysiol 1997; 103: 554-562.

23. Zigmond AS, Snaith RP. The Hospital Anxiety and Depression Scale. Acta Psychiatrica Scandinava 1983; 67: 361-370.

24. Perneger TV, Leplège A, Etter J-F, Rougemont A. Validation of a french-language version of the MOS 36-item short form health survey (SF-36) in young healthy adults. $J$ Clin Epidemiol 1995; 48: 1051-1060.

25. Ware JE, ed. SF-36 Health Survey. Manual and Interpretation Guide. The Health Institute, New England Medical Center. Boston, Nimrod Press, 1993.

26. Stepanski E, Lamphere J, Roehrs T, Zorick F, Roth T. Experimental sleep fragmentation in normal subjects. Int J Neurosci 1987; 33: 207-214.

27. Wesensten NJ, Balkin TJ, Belenki G. Does sleep fragmentation impact recuperation? A review and reanalysis. J Sleep Research 1999; 8: 237-245.

28. Chervin RD. Sleepiness, fatigue, tiredness and lack of energy in obstructive sleep apnea. Chest 2000; 118: 372-379.

29. Fontaine KR, Barofsky I. Obesity and health-related quality of life. Obes Rev 2002; 2: 173-182. 